\title{
Optimization of the ordinal and cardinal consistency of a preference matrix in decision making
}

\author{
Martin Gavalec ${ }^{1}$ Karel Mls $^{1}$ Hana Tomášková ${ }^{1}$ \\ ${ }^{1}$ University of Hradec Králové, Rokitanského 62, 50003 Hradec Králové, Czech Republic
}

\begin{abstract}
In multi-criteria decision problems the relative importance of alternatives is computed from preference matrices, which come from experience and can possibly be inconsistent. Two consistency types of preferences are studied in the paper. The ordinal consistency preserves the order in which the alternatives are arranged, and it does not allow cycles. The term 'cyclic consistency' is also used for this type. The second type is the cardinal consistency, when not only the order, but also the exact values of the relative importance must be consistent.

In this paper efficient algorithms for computing consistent approximations of both types for a given preference matrix are described. The main result is an algorithm which combines the advantages of both particular types and computes the optimal consistent approximation of a given preference matrix in the ordinal and in the cardinal sense. The described algorithm can also be used for processing preference matrices with missing data. The performance of the algorithm is illustrated by numerical examples.
\end{abstract}

Keywords: decision making, preference matrix, consistent preference matrix, ordinal consistency, cardinal consistency, consistent approximation, optimization, optimal consistent approximation

\section{Introduction}

The Analytical Hierarchy Process (AHP) formulated by [12] belongs to the basic methods developed for solving multi-criteria decision problems. The method has been applied in many cases, see e.g. $[14,15,9,11,3,7]$. Using subjective pairwise comparisons, numerical values are assigned to individual components of the model, showing their relative importance. One of the fundamental questions in AHP is how to find the appropriate preference matrix for a set of alternatives.

The preferences given by human experts are often inconsistent and do not reflect the deep relations between the processed notions, see $[2,8,1,10,11$, $6]$. By [13], the standard approach to the problem of finding the relative importance vector out of an inconsistent preference matrix uses an eigenvector of the preference matrix computed by the methods of linear algebra. With the help of this eigenvector, the so-called inconsistency index is computed, and the use of the preference matrix is recommended when the index value does not exceed the empirical value 0.1 . We can say that this method empirically estimates that the expert's preferences are 'not too inconsistent' for the practical purposes.

More exact direct methods for achieving the consistency of a given relative preference matrix have been suggested in $[4,5]$. The first method removes the so-called ordinal inconsistency which violates the order in which the alternatives are arranged, and creates cycles (cyclic inconsistency). The second method achieves the cardinal consistency, when not only the order, but also the exact values of the relative importance are optimized to be consistent. Efficient algorithms for computing consistent approximations of both types for a given preference matrix have been described.

A general approach involving the ordinal as well as the cardinal consistency is suggested in this paper. The algorithm works in two phases. In Phase 1, the inconsistent cycles are removed and the obtained preference matrix is then optimized by linear programming method to get the closest consistent matrix (consistent approximation) in Phase 2.

We use the additive form of expressing the relative importance in every pair of alternatives. It is shown that a matrix is cardinal consistent exactly when the diagonal elements are equal to zero, and all the columns are parallel, that is, when the difference of any pairs of columns is a constant vector. It is important to remark that a similar characterization of consistency is possible also in the multiplicative form of relative preference (taking ones instead of zeros and quotiens instead of differences). Both approaches are equivalent and can be easily transformed to each other. The additive form is used here because it is more convenient for the optimization purposes - the linear programming methods can directly be applied.

\section{Consistency of preference matrices}

In multicriterial decision making we work with a fixed set of alternatives $\mathcal{A}_{1}, \mathcal{A}_{2}, . ., \mathcal{A}_{n}$. Till the end of the paper $N$ will denote the set $\{1,2, \ldots, n\}$ and $\mathcal{R}$ the set of all real numbers. The quantified judg- 
ments on pairs $\mathcal{A}_{i}, \mathcal{A}_{j}$ are represented by an $n \times n$ matrix $A=\left(a_{i j}\right), i, j \in N$. Every entry $a_{i j}$ is real number, which is interpreted as an evaluation of the relative preference of $\mathcal{A}_{i}$ with respect to $\mathcal{A}_{j}$, in the additive sense. That is, $\mathcal{A}_{i}$ is considered to be by $a_{i j}$ better than $\mathcal{A}_{j}$. Conversely, $\mathcal{A}_{j}$ is better by $-a_{i j}$ than $\mathcal{A}_{i}$. In this interpretation, $a_{i i}=0$ for every $i \in N$. Then $A$ is called additive preference matrix (for short: preference matrix) of the alternatives $\mathcal{A}_{1}, \mathcal{A}_{2}, . ., \mathcal{A}_{n}$. The basic properties of the preference matrix sound as follows

$A$ is antisymmetric if $a_{i j}=-a_{j i}$ for every $i, j \in N$, $A$ is consistent if $a_{i j}+a_{j k}=a_{i k}$ for every $i, j, k \in N$.

Equivalent definitions are

$A$ is antisymmetric if $a_{i j}+a_{j i}=0$ for every $i, j \in N$, $A$ is consistent if $a_{i j}+a_{j k}+a_{k i}=0$ for every $i, j, k \in$ $N$.

It is easy to see that if $A$ is consistent, then $A$ is antisymmetric. On the other hand, the converse implication is not true. Clearly, $A$ is antisymmetric if and only if it is of the form

$$
A=\left(\begin{array}{cccc}
0 & a_{12} & \cdots & a_{1 n} \\
-a_{12} & 0 & \cdots & a_{2 n} \\
\cdots & \cdots & \cdots & \cdots \\
-a_{1 n} & -a_{2 n} & \cdots & 0
\end{array}\right)
$$

E.g., $A=\left(\begin{array}{rrr}0 & 1 & 1 \\ -1 & 0 & 1 \\ -1 & -1 & 0\end{array}\right)$ is antisymmetric, but it is not consistent, because $a_{12}+a_{23}=1+1=$ $2 \neq a_{13}$.

Remark 2.1 The consistency property defined above is sometimes called the cardinal consistency. Another consistency type, the ordinal consistency will be considered in Section 3.

In real applications, the preference matrix is created by an expert in the given field. While the antisymmetricity is easy to verify by formula (1), the consistency cannot be directly seen from the data.

Consistent matrices have been characterized in [5] by the following property. We say that vectors $x, y \in \mathcal{R}(n)$ are parallel, if there is $c \in \mathcal{R}$ such that $y_{i}=c+x_{i}$ for every $i \in N$ (we shortly write: $y=$ $c+x)$.

Theorem 2.2 [5] If all diagonal entries of a matrix $A$ are equal to zero, then the following statements are equivalent

(i) $A$ is consistent,

(ii) every two columns of A are parallel.

\section{Ordinal consistency of a preference matrix}

Preference matrix $A$ is called cyclic inconsistent, if there is a cycle

$$
i_{1} i_{2} \ldots i_{r-1} i_{r} i_{1}
$$

of length $r \geq 2$ of indices in $N$, called: inconsistent cycle in $A$, such that the inequalities

$$
a\left(i_{k} i_{k+1}\right) \geq 0 \quad \text { for every } \quad k=1,2, \ldots, r
$$

hold, and at least one of the inequalities is strict. Matrix $A$ is cyclic consistent, or ordinal consistent, if every cycle in $A$ is consistent, i.e. if there are no inconsistent cycles in $A$.

It has been shown in [4] that the above definition can be reduced to inconsistent cycles of lenth $r=3$, see examples of such cycles in Figure 1.

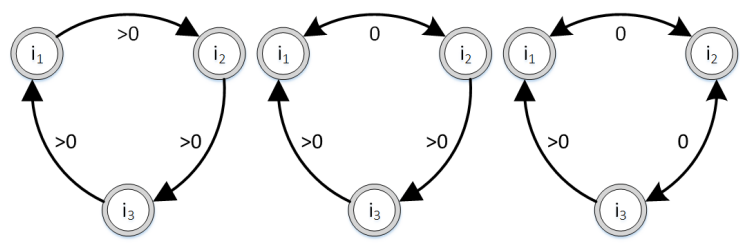

Figure 1: Possible types of cyclic inconsistency with $r=3$

Theorem 3.1 [4] If an antisymmetric matrix $A$ contains an inconsistent cycle of length $r>3$, then $A$ also contains an inconsistent cycle of length 3 .

For an inconsistent cycle of length 4, Theorem 3.1 is illustrated in Figure 2.

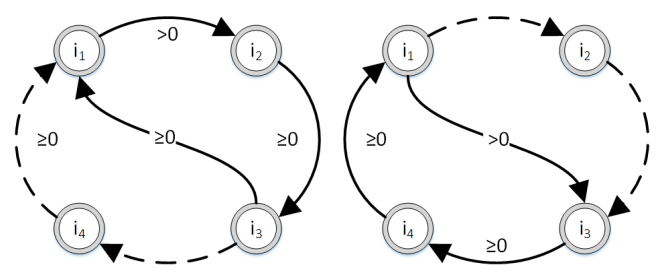

Figure 2: Cyclic inconsistency with $r=4$

Corollary 3.2 The cyclic consistency of a complete preference matrix can be recognized in time $O\left(n^{3}\right)$, by verifying all index cycles of length 3 for the inconsistency.

If the cyclic inconsistency of $A$ has been recognized (that is, at least one inconsistent cycle of length 3 has been found), then a natural way of treating the inconsistency is to change all entries in the inconsistent cycles to value 0 . In view of Corollary 3.2, one could suppose that it would be sufficient to process all index cycles of length 3 , one by one. However, this direct method does not work. It can happen that the procedure creates new inconsistent cycles which had not been previously present. For example, the inconsistent cycle $i_{1} i_{3} i_{4} i_{1}$ of length 3 in Figure 3 only appears after treating the cycle $i_{1} i_{2} i_{3} i_{1}$.

The above disadvantage is not present in another method described in [4], which works with the strongly connected components in the preference digraph $\mathcal{D}(A)$ (SCC method, for short). The method 


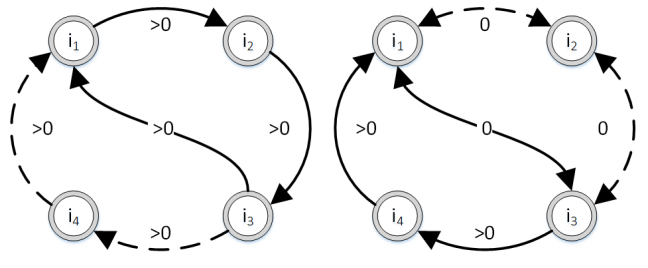

Figure 3: Creating new inconsistent cycles of lenth $r=3$

is based on Theorem 3.4 below. The preference $d i$ graph $\mathcal{D}(A)=(V(A), E(A))$ of $A$ which is defined as follows

$$
\begin{aligned}
& V(A)=\{1,2, \ldots, n\} \\
& E(A)=\left\{(i, j) ; a_{i j} \geq 0\right\} \\
& E^{+}(A)=\left\{(i, j) ; a_{i j}>0\right\}
\end{aligned}
$$

The edges in $E^{+}(\mathcal{D})$ are called positive preference edges.

Theorem 3.3 [4] Preference matrix $A$ is cyclic consistent if and only if every cycle $C$ in $\mathcal{D}(A)$ contains no positive preference edges, i.e. $C \cap E^{+}(A)=$ $\emptyset$.

Theorem 3.4 [4] Preference matrix $A$ is cyclic consistent if and only if every strongly connected component $\mathcal{K}$ in $\mathcal{D}(A)$ contains no positive preference edges, i.e. $(\mathcal{K} \times \mathcal{K}) \cap E^{+}(A)=\emptyset$.

The following algorithm for computing a cyclic consistent matrix approximating a given preference matrix $A$ is naturally motivated by Theorem 3.4 .

\section{SCC algorithm}

1 find all strongly connected components in the preference digraf $\mathcal{D}(A)$

2 change all relative preferences within the strongly connected components to 0

Theorem 3.5 [4] If matrix $A^{\prime}$ is created from an antisymmetric matrix $A$ by the SCC algorithm, i.e.

$a_{i j}^{\prime}= \begin{cases}0 & \text { for } i, j \text { in some strongly connected } \\ a_{i j} & \text { otherwise }\end{cases}$

then $A^{\prime}$ is antisymmetric and cyclic consistent.

The cyclic consistent matrix $A^{\prime}$ computed by the $\mathrm{SCC}$ algorithm is called the ordinal consistent approximation of $A$.

\section{Cardinal consistent approximation}

Consider the following optimization problem: given a matrix $A$ (which may be inconsistent), find a consistent matrix $\tilde{A}$ which will be as close to $A$ as possible. Clearly, if $A$ is consistent, then $\tilde{A}=A$. Matrix
$\tilde{A}$ is called the cardinal consistent approximation of $A$ (see also [5]).

The terminology in [5] slightly differs from that used in this paper. For the convenience of the reader we present the results with complete proofs.

A given consistent preference matrix is related to a vector showing the importance of the alternatives. In the additive notation, $w \in \mathcal{R}(n)$ is called a balanced vector if $\sum_{i \in N} w_{i}=0$. When alternatives $\mathcal{A}_{1}, \mathcal{A}_{2}, \ldots, \mathcal{A}_{n}$ are considered, then $w_{i}$ is interpreted as the weight of $\mathcal{A}_{i}$ for every $i \in N$. The differences of weights are the entries of the corresponding matrix of relative preferences $A(w)$ with $a_{i j}(w)=w_{i}-w_{j}$ for $i, j \in N$. We say that $w i n$ duces $A(w)(A(w)$ is induced by $w)$.

\section{Theorem 4.1 [5]}

(i) If $w \in \mathcal{R}(n)$, then $A(w)$ is consistent.

(ii) If $A$ is a consistent matrix, then there is a unique balanced vector $w$ inducing $A$.

Proof: (i) If $w \in \mathcal{R}(n)$ is a weight vector, then $a_{i j}(w)+a_{j k}(w)+a_{k i}(w)=\left(w_{i}-w_{j}\right)+\left(w_{j}-w_{k}\right)+$ $\left(w_{k}-w_{i}\right)=0$ for every $i, j, k \in N$. That is, $A(w)$ is consistent.

(ii) Assume that $A \in \mathcal{R}(n, n)$ is consistent. Define $w \in \mathcal{R}(n)$ by putting

$$
w_{i}=\frac{1}{n} \sum_{k \in N} a_{i k} \quad \text { for every } i \in N .
$$

Using the consistency assumption in computation

$$
\begin{aligned}
\sum_{i \in N} w_{i} & =\frac{1}{n} \sum_{i \in N} \sum_{k \in N} a_{i k} \\
& =\frac{1}{n}\left(\sum_{i \in N} a_{i i}+\sum_{\{i, k\} \subseteq N, i \neq k}\left(a_{i k}+a_{k i}\right)\right) \\
& =\frac{1}{n}(0+0)=0,
\end{aligned}
$$

we verify that $w$ is a balanced vector. By further computation we get, for every $i, j \in N$,

$$
\begin{aligned}
a_{i j}(w) & =w_{i}-w_{j}=\frac{1}{n} \sum_{k \in N} a_{i k}-\frac{1}{n} \sum_{k \in N} a_{j k} \\
& =\frac{1}{n} \sum_{k \in N} a_{i j}=a_{i j}=\frac{1}{n} \sum_{k \in N}\left(a_{i k}+a_{k j}\right)
\end{aligned}
$$

that is, $A(w)=A$. The uniqueness of $w$ then follows directly from $(3)$.

In view of Theorem 4.1, the closest consistent matrix to a given preference matrix $A$ can be found by looking for a suitable balanced vector $w$ such that the distance between $A$ and $A(w)$ will be as small as possible. The distance $d$ will be measured by the well-known formula

$$
d(A, A(w))=\max _{i, j \in N}\left|a_{i j}-a_{i j}(w)\right| .
$$


Theorem 4.2 [5] If $A$ is antisymmetric, then

$$
d(A, A(w))=\max _{i, j \in N}\left(a_{i j}-w_{i}+w_{j}\right) .
$$

Proof: Assume that $A$ is antisymmetric. Then the following inequalities are equivalent for any $i, j \in N$, $m \in \mathcal{R}$

$$
\begin{aligned}
& \left|a_{i j}-a_{i j}(w)\right| \leq m \\
\Leftrightarrow & \left|a_{i j}-w_{i}+w_{j}\right| \leq m \\
\Leftrightarrow & \left(-m \leq a_{i j}-w_{i}+w_{j}\right) \wedge\left(a_{i j}-w_{i}+w_{j} \leq m\right) \\
\Leftrightarrow & \left(m \geq-a_{i j}+w_{i}-w_{j}\right) \wedge\left(a_{i j}-w_{i}+w_{j} \leq m\right) \\
\Leftrightarrow & \left(a_{j i}-w_{j}+w_{i} \leq m\right) \wedge\left(a_{i j}-w_{i}+w_{j} \leq m\right)
\end{aligned}
$$

Clearly, (4) is an immediate consequence of (5).

Computing the best consistent approximation of a preference matrix given by an expert can be formulated as the following minimization problem.

OCA (optimal consistent approximation) Input: antisymmetric matrix $A \in \mathcal{R}(n, n)$ Variables: $m \in \mathcal{R}, w=\left(w_{1}, w_{2}, \ldots, w_{n}\right) \in \mathcal{R}(n)$ minimize

$$
z=m \longrightarrow \min
$$

subject to

$$
\begin{gathered}
\sum_{i \in N} w_{i}=0 \\
m+w_{i}-w_{j} \geq a_{i j} \quad \text { for } i, j \in N .
\end{gathered}
$$

$\mathcal{S}(A)$ denotes the set of all optimal solutions to the OCA problem with input $A$.

Theorem 4.3 [5] If $A$ is antisymmetric and $(m, w) \in \mathcal{S}(A)$, then

(i) $A$ is consistent if and only if $m=0$,

(ii) $m$ is the minimal possible distance of a consistent matrix to $A$,

(iii) $w$ is a balanced vector and the induced matrix $A(w)$ is the nearest consistent approximation of $A$ with $d(A, A(w))=m$.

Proof: (iii) Assume that $A$ is antisymmetric and $(m, w) \in \mathcal{S}(A)$. Then $w$ is a balanced vector in view of $(6)$. The following inequalities are equivalent for any $i, j \in N$

$$
m+w_{i}-w_{j} \geq a_{i j} \quad \Leftrightarrow \quad a_{i j}-w_{i}+w_{j} \leq m .
$$

In view of (7) and (8), $\max _{i, j \in N}\left(a_{i j}-w_{i}+w_{j}\right) \leq m$. Therefore, $d(A, A(w)) \leq m$, by Theorem 4.2. The equality $d(A, A(w))=m$ follows by the minimality of $m$.

(i), (ii) Assertion (ii) follows from (iii) and from Theorems 4.1, 4.2. Finally, assertion (i) follows from (ii).
Example 1: Consider the antisymmetric preference matrix

$$
A=\left(\begin{array}{rrrr}
0 & 2 & 4 & 8 \\
-2 & 0 & 4 & 6 \\
-4 & -4 & 0 & 2 \\
-8 & -6 & -2 & 0
\end{array}\right)
$$

$A$ is inconsistent, because $a_{12}+a_{23}=2+4=6 \neq$ $a_{13}$. The minimization problem OCA with input $A$ has the solution $(m, w)$, where $m=0.66$ and

$$
w=\left(\begin{array}{r}
3.33 \\
2 \\
-1.33 \\
-4
\end{array}\right)
$$

is the balanced weight vector of the four considered alternatives represented by the columns of $A$. The induced matrix

$$
A(w)=\left(\begin{array}{rrrr}
0 & 1.33 & 4.66 & 7.33 \\
-1.33 & 0 & 3.33 & 6 \\
-4.66 & -3.33 & 0 & 2.66 \\
-7.33 & -6 & -2.66 & 0
\end{array}\right)
$$

is the optimal consistent approximation of $A$ with the minimal distance $m=d(A, A(w))=0.66$.

\section{Optimal consistent preferences}

In the previous two sections, the ordinal and cardinal approach to the possible inconsistency of a preference matrix $A$ created by an expert are described. Both approaches are merged in this section. An algorithm working in two phases is described.

First, the optimal cyclic consistent approximation $A^{\prime}$ of $A$ is computed. In this phase, the pairs of alternatives are found which have the same preference ranking, by the expert's opinion. Thus, $A^{\prime}$ describes an equivalence relation on the set of all considered alternatives.

In the second phase, $A^{\prime}$ is further optimized by the linear optimization OCA in such a way that the equivalence relation previously computed will be preserved.

Preference pre-order $\mathcal{P}(A)$ induced by $A$ is defined as follows: if inequalities $a\left(i_{k} i_{k+1}\right) \geq 0$ with $k=1,2, \ldots, r-1$ hold for some sequence $i=$ $i_{1}, i_{2}, \ldots, i_{r}=j$, then $(i, j) \in \mathcal{P}(A)$, i.e. alternative $A_{i}$ has higher (or equal) preference ranking than $A_{j}$.

Remark 5.1 The pre-order relation $\mathcal{P}(A)$ is reflexive and transitive. In general, $\mathcal{P}(A)$ is not antisymmetric, if two different alternatives have the same ranking (are equivalent).

Theorem 5.2 If matrix $A$ is cyclic consistent, then $\mathcal{P}(A)$ is a uniquely determined linear pre-order of alternatives, up to permutations of equivalent alternatives. 
PROOF: The assertion follows from the definition of the cyclic consistency and that of the preference pre-order $\mathcal{P}(A)$.

If $A \in \mathcal{R}(n, n)$ is cyclic consistent, then two alternatives $\mathcal{A}_{i}, \mathcal{A}_{j}$ are equivalent if and only if the corresponding nodes $i, j$ belong to the same strongly connected component in $\mathcal{D}(A)$. The equivalent alternatives should have the same relative preference to any other alternative. This is the basic idea of the combined algorithm $\mathrm{CA}$ below.

For $A \in \mathcal{R}(n, n)$, the set of all strongly connected components in $\mathcal{D}(A)$ will be denoted by $\operatorname{SCC}(A)$.

\section{CA algorithm}

1 Input: antisymmetric matrix $A \in \mathcal{R}(n, n)$

2 Compute preference digraph $\mathcal{D}(A)$

3 Compute cyclic consistent approximation $A^{\prime}$ of $A$ by SCC algorithm

4 For every pair $\mathcal{K}, \mathcal{L} \in \operatorname{SCC}(A)$ substitute all values $a_{i j}^{\prime}$ with $i \in \mathcal{K}, j \in \mathcal{L}$ by their common arithmetic mean $a^{\prime \prime}{ }_{i j}=\frac{1}{n} \sum_{i \in \mathcal{K}, j \in \mathcal{L}} a^{\prime}{ }_{i j}$

5 Compute the optimal consistent approximation $\tilde{A}$ of $A^{\prime \prime}$ by linear optimization OCA with additional constraints $w_{i}-w_{j}=0$ for every $i, j \in \mathcal{K}$, $\mathcal{K} \in \operatorname{SCC}(A)$

6 Output: antisymmetric and consistent approximation matrix $\tilde{A}$ with $\mathcal{P}\left(A^{\prime}\right)=\mathcal{P}(\tilde{A})$

Theorem 5.3 CA algorithm works correctly and for every $n \times n$ antisymmetric preference matrix $A$ the algorithm computes a consistent aproximation $\tilde{A}$ preserving the order of the alternatives, in $O\left(n^{2}\right)$ time.

PROOF: The correctness of the algorithm follows from Theorem 5.2. The computation of the strongly connected components and their order in the preference digraph $\mathcal{D}(A)$ can be done by the depth-first algorithm in $O\left(n^{2}\right)$ time, as well as the rest of the computation.

Remark 5.4 CA algorithm can easily be modified also for the case of missing values in the input preference matrix $A$. In the modification, the missing input values create no edges in the preference $d i$ graph $\mathcal{D}(A)$.

Example 2: Consider the antisymmetric preference matrix $A$ as the input in $\mathrm{CA}$ algorithm

$$
A=\left(\begin{array}{rrrrrr}
0 & 1 & -1 & 1 & 2 & 2 \\
-1 & 0 & 1 & 1 & 2 & 3 \\
1 & -1 & 0 & 1 & 1 & 2 \\
-1 & -1 & -1 & 0 & 1 & 1 \\
-2 & -2 & -1 & -1 & 0 & 1 \\
-2 & -3 & -2 & -1 & -1 & 0
\end{array}\right)
$$

The computation in step 2 finds a single inconsistent cycle $(1,2,3,1)$ with $a_{12}+a_{23}+a_{31}=1+1+1>$
0 in $A$. That is, the strongly connected components of $\mathcal{D}(A)$ are $\mathcal{K}_{123}=\{1,2,3\}, \mathcal{K}_{4}=\{4\}, \mathcal{K}_{5}=\{5\}$ and $\mathcal{K}_{6}=\{6\}$.

The cyclic consistent approximations $A^{\prime}, A^{\prime \prime}$ are computed in steps 3,4 .

$$
A^{\prime}=\left(\begin{array}{rrrrrr}
0 & 0 & 0 & 1 & 2 & 2 \\
0 & 0 & 0 & 1 & 2 & 3 \\
0 & 0 & 0 & 1 & 1 & 2 \\
-1 & -1 & -1 & 0 & 1 & 1 \\
-2 & -2 & -1 & -1 & 0 & 1 \\
-2 & -3 & -2 & -1 & -1 & 0
\end{array}\right)
$$

$$
\begin{gathered}
A^{\prime \prime}= \\
\left(\begin{array}{rrrrrr}
0 & 0 & 0 & 1 & 1,67 & 2,33 \\
0 & 0 & 0 & 1 & 1,67 & 2,33 \\
0 & 0 & 0 & 1 & 1,67 & 2,33 \\
-1 & -1 & -1 & 0 & 1 & 1 \\
-1,67 & -1,67 & -1,67 & -1 & 0 & 1 \\
-2,33 & -2,33 & -2,33 & -1 & -1 & 0
\end{array}\right) .
\end{gathered}
$$

The output matrix $\tilde{A}$ is computed from $A^{\prime \prime}$ by linear optimization OCA with additional constraints in step 5 .

$$
\begin{gathered}
\tilde{A}= \\
\left(\begin{array}{rrrrrr}
0 & 0 & 0 & 0,89 & 1,56 & 2,22 \\
0 & 0 & 0 & 0,89 & 1,56 & 2,22 \\
0 & 0 & 0 & 0,89 & 1,56 & 2,22 \\
-0,89 & -0,89 & -0,89 & 0 & 0,67 & 1,33 \\
-1,56 & -1,56 & -1,56 & -0,67 & 0 & 0,67 \\
-2,22 & -2,22 & -2,22 & -1,33 & -0,67 & 0
\end{array}\right)
\end{gathered}
$$

\section{Conclusions}

Two consistency types of relative ranking of alternatives in a multi-criteria decision problem have been studied. The ordinal consistency (cyclic consistency) studies the order in which the alternatives are arranged, and does not allow cycles. The cardinal consistency demands that not only the order of alternatives, but also the values of the relative importance are consistent.

Efficient algorithms for computing consistent approximations of both types for a given preference matrix are described. The main result is a merged algorithm computing the optimal consistent approximation of a given preference matrix in both the ordinal and the cardinal sense. The described algorithms can also be used for processing preference matrices with missing data. The work of the presented methods is illustrated by examples.

\section{Acknowledgement}

The support of Czech Science Foundation GAČR \#14-02424S and the grant project UHK FIM SPEV \# 2106 is gratefully acknowledged. 


\section{References}

[1] Aguarón, J., Escobar, M. T., Moreno-Jiménez, J.M. (2002). Consistency stability intervals for a judgement in AHP decision support systems, in: European Journal of Operational Research, vol. 145, 2002.

[2] Carlsson, C., Fullér, R. (1996). Fuzzy multiple criteria decision making: Recent developments, Fuzzy Sets and Systems, Volume 78, Issue 2, 11 March 1996, Pages 139-153.

[3] Dytczak, M., Szklennik, N. (2011). Principles and applications of AHP/ANP - based multiple $M C D A$ methods approach, in: ISAHP 2011. Proceedings, Sorrento, 15-18 June 2011.

[4] Gavalec, M., Mls, K.(2014). Consistency of expert-based preference matrices, in: Proceedings ISAHP 2014. Washington, 29 June - 7 July 2014. Pages 1-11.

[5] Gavalec, M., Tomášková (2014). Consistent approximation of preference matrices and maxprod eigenvectors, Int. J. Mathematics in OR (submitted).

[6] Gavalec, M., Ramík, J. \& Zimmermann, K. (2014). Decision Making and Optimization, Special Matrices and Their Applications in Economics and Management, Lecture Notes in Econom. and Math. Systems 677, SpringerVerlag Berlin, 2014.

[7] Jalaob, E.R., Wua, T., Shunka D. (2014). A stochastic AHP decision making methodology for imprecise preferences, Information Sciences 270 (2014), 192-203.

[8] Leung, L.C., Cao, D. (2000). On consistency and ranking of alternatives in fuzzy AHP, European Journal of Operational Research, Volume 124, Issue 1, 1 July 2000, Pages 102-113.

[9] Mls, K. and Gavalec, M. (2009). Multi-criteria models in autonomous decision making systems. In Proceedings of the 10th International Symposium on the Analytic Hierarchy/Network Process. pp 1-8, University of Pittsburgh, Pittsburgh, Pennsylvania, USA.

[10] Ramík, J., Korviny, P. (2010). Inconsistency of pair-wise comparison matrix with fuzzy elements based on geometric mean, Internat. J. Fuzzy Sets and Systems 161(2010), 1604-1613.

[11] Ramík, J., Perzina, R. (2010). A method for solving fuzzy multicriteria decision problems with dependent criteria, Fuzzy Optimization and Decision Making, Springer Verlag,Vol.9, No.2, 123-141.

[12] Saaty, T.L. (1980). The analytic hierarchy process. McGraw-Hill, New York.

[13] Saaty, T.L. (2003). Decision-making with the AHP: Why is the principal eigenvector necessary, European Journal of Operational Research, Volume 145, Issue 1, 16 February 2003, Pages 85-91.

[14] Vargas, L. (1982). Reciprocal matrices with random coefficients, Mathematical Modelling, Volume 3 (1982), 69-81.

[15] Zahedi, F. (1986). The Analytic Hierarchy Process: A Survey of the Method and Its Applications,Interfaces, Volume 16 (1986), 96-108. 